\title{
Zählung von Elementarteilchen und Quanten mit Elektronenvervielfacher und Leuchtschirm*
}

\author{
Von Ewald Fünfer ${ }^{* *}$ \\ (Z. Naturforschg. 4a, 672-682 [1949]; eingegangen am 4. März 19.19)
}

\begin{abstract}
Es werden die Eigenschaften eines Leuchtschirm-Zählers, bestehend aus einem Elektronenvervielfacher (Type I P 21) und Leuchtschirm untersucht. Zur Zählung von $\alpha$-Strahlen erweist sich ein Zinksulfid-Leuchtschirm als geeignet; $\alpha$-Strahlen von einer gewissen Energie ab werden mit einem Wirkungsgrad von $100 \%$ gezählt. Für Radium $\beta$ - und $\gamma$-Strahlung werden nach Kallmann Naphthalin- und Anthracen-Leuchtschirme von mehreren mm Dicke verwendet. Mit Kühlung erreicht man damit die 10-fache Ausbeute an $\gamma$-Strahlung wie beim Zählrohr. Durch Oszillogramme der Vervielfacherimpulse wird eine direkte Bestimmung der z. B. für Koinzidenzmessungen wichtigen Impulsdauer durchgeführt. Für Anthracen ergibt sich eine Impulsdauer von $1^{-7}$ sec. Die Amplituden der vom Vervielfacher gelieferten Impulse zeigen eine sehr große Streuung. An Hand einiger Messungen wird gezeigt, daß diese Verteilungsfunktion einen Rückschluß auf die am Eingang des Vervielfachers startende Zahl von Elektronen und damit auf die im Leuchtschirm absorbierte Strahlungsenergie gestattet.
\end{abstract}

$\mathrm{B}$ a $y^{1}$, Allen ${ }^{2}, M_{\text {eyer }}^{3}$ u. a. haben gezeigt, daß es möglich ist, mit dem Elektronenvervielfacher Elementarteilchen und Quanten zu zählen. Man läßt bei diesem Verfahren die zu messende Strahlung auf die erste Sekundärelektrode bzw. Photokathode des Vervielfachers fallen, so daß darin einige Sekundärelektronen ausgelöst werden. Die Zahl dieser Elektronen hängt von der einfallenden Strahlung ab, sie ist im Fall des Photoeffektes, bei sichtbarem Licht etwa, gleich 1 und steigt bei $\alpha$-Strahlen bis etwa 10 an. Die dadurch am Ausgang des Vervielfachers erzeugten Impulse liegen bei Zimmertemperatur nicht wesentlich über den statistischen Schwankungen des Dunkelstromes, welcher durch die thermische Emission der Sekundärelektroden entsteht. Es ist deshalb meistens eine Kühlung des Vervielfachers erforderlich, um die thermische Emission zu beseitigen. Falls die Energie der zu messenden Lichtquanten dies zuläßt, kann an Stelle der Kühlung die Austrittsarbeit der Sekundärschichten erhöht

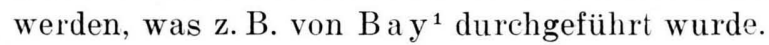

Der wesentliche Fortschritt eines solchen Zählers gegenüber dem Geiger-Müller-Zählrohr liegt in der außerordentlich kurzen Impulsdauer, für die z. B. Papp ${ }^{4}$ bei seinen Messungen den Wert

* Vorgetragen auf der Physikertagung in Heidelberg am 30. u. 31. Jan. 1949.

** (17 b) Weil (Rhein), Gartenstr. 2.

1 Z. B a y, Z. Physik 3/4, 227 [1941].

2 I. S. All e n, Physic. Rev. 61, 692 [1942].

3 K. P. M e y e r, Helv. physica Acta 19, 211 [1946].
$5 \cdot 10^{-9}$ sec erhielt. Man kann also sehr große Impulszahlen pro sec messen. Während beim Geiger-Müller-Zählrohr nach jedem Impuls eine gewisse Zeit vergehen muß, bevor das Zählrohr wieder meßbereit ist, existiert eine solche Totzeit beim Vervielfacher nicht. Er kann infolgedessen bei steigender Impulshäufigkeit zuerst als Zähler und dann als kontinuierliches Strahlungsmeßgerät dienen. Benützt man den Vervielfacher zu Koinzidenzmessungen, so erhält man ein um mehrere Größenordnungen besseres Auflösungsvermögen als mit dem Geiger-Müller-Zählrohr. Als Grenze für das Auflösungsvermögen wurden $10^{-9}$ bis $10^{-10}$ sec angegeben. Um dieses hohe Auflösungsvermögen ausnützen zu können, sind spezielle Koinzidenzanordnungen nötig, wie sie verschiedentlich entwickelt wurden ${ }^{5,6}$.

Zur Zählung energiereicher Teilchen und Quanten läßt sich in vielen Fällen vorteilhafter eine Kombination von Vervielfacher und Leuchtschirm benutzen. Die zu messende Strahlung erzeugt dabei in einem geeigneten Leuchtschirm eine Lumineszenzstrahlung, die beim Auftreffen auf die Photokathode des Vervielfachers Elektronen auslöst. Die Zahl dieser Elektronen pro Teilchen der zu messenden Strahlung ist dabei in vielen Fällen wesentlich größer, als wenn das

${ }^{4}$ G. P a p p, Rev. sci. Instruments 19, 568 [1948].

5 K. P. M e y er, P. Huber u. E. B ald inger, Helv. physica Acta 21, 188 [1948].

6 Z. B a y u. G. P a p p, Rev. sci. Instruments 19 , 565 [1948]. 
Teilchen direkt durch Sekundäremission an der Eingangselektrode des Vervielfachers Elektronen erzeugt hätte. Infolgedessen erhält man wesentlich größere Ausgangsimpulse am Vervielfacher, die zum großen Teil über den statistischen Schwankungen des Dunkelstromes liegen. Man kann also u. U. auf die lästige Kühlung des Vervielfachers verzichten. Mit diesem Verfahren lassen sich sehr leicht die in Zinksulfid- oder CalciumwolframatLeuchtschirmen von energiereichen $\alpha$-Strahlen erzeugten Szintillationen messen ${ }^{7,8}$. Die Zählung von Neutronen mit Zinksulfid-Leuchtschirmen läßt sich dadurch ermöglichen, daß man dem Zinksulfid Bor oder Lithium beimischt. Die bei den Reaktionen B (n, $\alpha)$ und $\mathrm{Li}(\mathrm{n}, \alpha)$ entstehenden $\alpha$-Strahlen lassen sich dann leicht zählen ${ }^{9}$.

Man hat den Zinksulfid-Leuchtschirm auch zur Zählung von schnellen Elektronen, Röntgenstrahlen und $\gamma$-Strahlen benutzt ${ }^{10,11}$. Da die Ausbeute an Lumineszenzstrahlung hierbei jedoch gering ist, muß eine außerordentlich sorgfältige Ausnützung des erzeugten Lichtes durch speziell ausgesuchte Vervielfacher vorgenommen werden. wenn man ohne Kühlung Impulse erhalten will, die über dem Dunkelrauschen liegen. Einen wesentlichen Fortschritt bei der Zählung von energiereichen Elektronen und Quanten brachten die Arbeiten von Kallmann und Mitarbb. ${ }^{12,13}$. Sie benutzten an Stelle des Zinksulfid-Leuchtschirmes organische Leuchtsubstanzen, und zwar erwies sich Naphthalin als besonders geeignete Substanz zur Zählung von energiereichen Elektronen und Quanten, während für $\alpha$-Strahlen Zinksulfid überlegen war. Von verschiedenen Seiten ${ }^{14-17}$ wurde ebenfalls mit Naphthalin und einer Reihe anderer ähnlicher Substanzen gearbeitet, so z. B. mit Anthracen, Stilben, Phenanthren usw. Die Erfolge, welche mit diesen Substanzen gegenüber Zinksulfid erzielt wurden, beruhen vor allem darauf, daß sie in sehr dicken Schichten noch relátiv wenig von dem erzeugten Fluoreszenzlicht. absorbieren, während dagegen die zu messende Primärstrahlung bereits verhältnismäßig stark absorbiert wird. Obgleich also vielleicht sogar

7 I. B roser u. H. K allmann, Z. Naturforschg. 2 a, 439 [1947].

S. C. Curran u. W. R. B a k e r, Rev. sci. Instruments 19, 116 [1948].

${ }^{9}$ Th. Kah an, J. D e b i esse, R. Champeix u. H. B i z ot, J. Physique Radium 8, 25 [1948].

10 F. H. M a rshall, J. W. Coltman u. A. I. B e n n e t, Rev. sci. Instruments 19, 744 [1948].

11 R. I. M o o n, Physic. Rev. 73, 1210 [1948]. die Ausbeute an Fluoreszenzlicht, auf gleiche Schichtdicken der Leuchtschirmsubstanz bezogen, für diese Stoffe schlechter ist als für Zinksulfid, ist doch infolge der wesentlich größeren Schichtdicke die Gesamtausbeute höher.

Das Ziel der vorliegenden Arbeit ist eine weitere Klärung der Erscheinungen, welche beim Arbeiten mit derartigen Leuchtschirm-Zählern auftreten. Zur Zählung von $\alpha$-Strahlen wurden Zinksulfid-Leuchtschirme benutzt, für $\beta$ - und $\gamma$ Strahlen Naphthalin- odér Anthracen-Leuchtschirme.

\section{M e $ß$ methode}

Die Zinksulfid-Leuchtschirme zur Zählung von $\alpha$-Strahlen wurden hauptsächlich nach zwei Gesichtspunkten ausgewählt. Die Nachleuchtdauer soll so gering wie möglich sein, und die Wellenlänge des emittierten Lichtes soll möglichst weitgehend mit dem Empfindlichkeitsmaximum der Photokathode des Vervielfachers zusammenfallen. Zur Zählung von $\beta$ - und $\gamma$-Strahlung wurden Naphthalin und Anthracen benutzt. Die betreffende Substanz wurde durch mehrfaches Umkristallisieren gereinigt, wodurch gegenüber der ungereinigten Substanz eine wesentliche Steigerung der Ausbeute festzustellen war. Die gereinigte Substanz wurde unter hohem Druck zu Leuchtschirmen verschiedener Dicke gepreßt. Abb. 1 zeigt eine Anordnung von Leuchtschirm und Vervieliacher, wie sie zur Zählung benutzt wurde. Der Leuchtschirm befindet sich unmittelbar auf der äußeren Glaswand des Vervielfachers vor der innen befindlichen Photokathode. Um eine möglichst weitgehende Ausnützung des Fluoreszenzlichtes zu erreichen, kann man nach dem Vorgang von Ma rsh a $11^{\mathbf{1 0}}$ einen Hohlspiegel anbringen, der in der Mitte durchbohrt ist, um die $\alpha$-Strahlung durchzulassen (Abb.1 a). Für die Zählung von $\beta$ - und $\gamma$-Strahlung mit Naphthalin- oder Anthracen-Leuchtschirmen größerer Dicke bringt der Hohlspiegel keine merkbaren Vorteile. Diese Leuchtschirme wurden deshalb bei den folgenden Versuchen so dicht wie möglich auf den Vervielfacher aufgebracht, jedoch ohne Hohlspiegel (Abb.1b). Die Größe der Leuchtschirme ist durch die Größe der Photokathode begrenzt, sie beträgt etwa $2 \mathrm{~cm}^{2}$. Um größere Leuchtschirme auszunützen, müßte mit einer entsprechenden Optik ein verkleinertes Bild des Leuchtschirms auf die Photokathode entworfen werden. Im Gegensatz zum Geiger-Müller-Zählrohr ist

12 J. B r o s e r u. H. K a $11 \mathrm{~m}$ a $\mathrm{n}$, Z. Naturforschg. 2 a, 642 [1947].

13 J. B roser, L. Herforth, H. K a ll man u. U. M. Martius, Z. Naturforschg. 3a, 6 [1948].

${ }_{14}$ M. D e uts ch, Nucleonics 2, ว8 [1948].

15 G. B. Collins u. R. C. Hoyt, Physic. Rev. 73, 1259 [1948].

${ }_{16}^{16}$ L. F. W o u te rs, Physic. Rev. 74, 489 [1948].

${ }_{17}$ H. T. Gittings, R. F. Tas che k, A. R. Ronzio, E. Jones u. W. J. Masilun, Physic. Rev. 75, 205 [1949]. 
also die Vergrößerung des Zählvolumens mit Schwierigkeiten verbunden, während die Definition eines bestimmten Winkelbereiches, innerhalb dessen eine Strahlung gemessen werden soll, und die Bestimmung von Reichweiten usw. mit den Leuchtschirmen genauer durchzuführen sind.

Als Vervielfacher wurden die drei Typen I P 21, I P 28 und $931 \mathrm{~A}$ der R.C.A. benutzt. Bei I P 21 und 931 A lieǵt das Maximum der spektralen Empfindlichkeit etwa bei $4200 \AA$ A. Zum UV hin begrenzt das Glasgehäuse die Empfindlichkeit, während der mit einem Spezialglas gebaute I P 28 bis etwa 2000 A empfindlich ist. Alle drei Vervielfacher enthalten 9 Sekundärstufen und sind elektrostatisch fokussiert. Der Verstärkungsgrad beträgt bei $100 \mathrm{~V} /$ Stufe etwa $2 \cdot 10^{6}$ für .I P $21,2 \cdot 10^{5}$ für I P 28 und $1 \cdot 10^{6}$ für $931 \mathrm{~A}$. Der Innen-

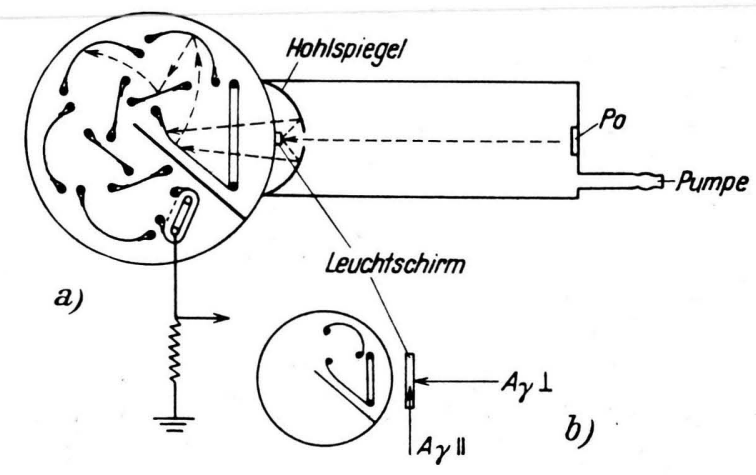

Abb. 1. Anordnung von Vervielfacher und Leuchtschirm: a) mit Hohlspiegel und Zinksulfid für $\alpha$ Strahlung, b) mit Anthracen für $\beta$ - und $\gamma$-Strahlung.

aufbau der Elektroden ist schematisch in Abb. $1 \mathrm{zu}$ sehen. Beim Vervielfacher existiert kein konstanter Zählbereich wie beim Geiger-Müller-Zählrohr, sondern die Zahl der gezählten lmpulse ändert sich sehr stark mit der Spannung am Vervielfacher. Infolgedessen muß die Hochspannung in Höhe von etwa $1000 \mathrm{~V}$ auf etwa $1 \%$ konstant gehalten werden. Die Unterteilung auf die einzelnen Stufen von je etwa $100 \mathrm{~V}$ geschieht mit einem hochohmigen Spannungsteiler. Da insbesondere in den letzten Stufen des Vervielfachers bei den sehr kurz dauernden Impulsen schon beträchtliche Spitzenströme auftreten, sind diese durch Kondensatoren ïberbrückt. Der durch thermische Emission bedingte Dunkelstrom war bei den einzelnen Vervielfachern stark verschieden. Füi die Versuche wurde eine Röhre mit möglichst kleinem Dunkelstrom ausgewählt, und zwar erwies sich ein ausgesuchter Vervielfacher I P 21 als besonders geeignet. Ohne Kühlung, also bei einer Temperatur von etwa $20^{\circ} \mathrm{C}$, war der Dunkelstrom etwa $10^{-7} \mathrm{~A}$, bei Kühlung mit flüssiger Luft unmeßbar klein. Bei Zimmertemperatur hat man also bereits eine recht große Zahl von Impulsen, größenordnungsmäßig $10^{5}$ his $10^{6} / \mathrm{sec}$, zu erwarten, die von thermischen Elektronen herrühren und sich den Impulsen der zu messen- den Strahlung überlagern. Da die störimpulse jedoch von je einem thermischen Elektron im Eingang des Vervielfachers erzeugt worden sind, die Meßimpulse aber mehreren Eingangselektronen entsprechen, unterscheiden sie sich durch ihre Amplituden. Dies gilt allerdings nur dann, wenn das Auflösungsvermögen des nachfolgenden Verstärkers sa hoch ist, daß nicht mit merklicher Häufigkeit mehrere thermische Elektronen sich überlagern können. Die Wahrscheinlichkeit $w_{n}$, dâs $n$ thermische Elektronen innerhalb des eben nicht mehr aufgelösten Zeitintervalls $\tau$ des Verstärkers auftreten, ist bei einer Impulshäufigkeit von v. Elektronen/sec gegeben durch ${ }^{\mathbf{1 8}}$

$$
w_{n}=\frac{(\nu \tau)^{n}}{n !} e^{-v \tau} .
$$

Daraus ergiht sich die Zahl $Z_{n}$ der Impulse/sec, welche von $n$ und mehr überlagerten thermischen Eingang :elektronen herrïhren zu

$$
Z_{n}=\frac{1}{\tau} \sum_{n}^{\infty} w_{n} .
$$

Ist die Zahl der zu messenden Impulse $a_{n} / \mathrm{sec}$, wobei im Mittel jeder Impuls durch $n$ Anfangselektronen erzeugt wird, so ist die erzielbare Meßgenauigkeit gegeben durch das Verhältnis der Meßimpulse $a_{n}$ zur Zahl der Störimpulse $Z_{n}$. Diese Betrachtung ist allerdings nur dann richtig, wenn Impulsdauer und Impulsform für Meß- und Störimpulse gleich sind. Die Dauer eines von einem einzigen Anfangselektron erzeugten Impulses ist etwa $10^{-\boldsymbol{9}} \mathrm{sec}$. Die Dauer der von Leuchtschirmszintillationen hervorgebrachten Impulse ist. wie später gezeigt wird, wesentlich länger. Die Dauer der von überlagerten thermischen Elektronen erzeugten Störimpulse kann jeden Zwischenwert annehmen. Außerdem sind auch die Impulsformen verschieden. Unter diesen Umständen ist das Verhältnis der gezählten Meßimpulse zur Zahl der Störimpulse auch noch ron den Eigenschaften des Verstärkers. insbesondere seiner Bandbreite, abhängig. Die obere Grenzfrequenz des an den Vervielfacher angeschlossenen Verstärkers entsprach mit $7 \mathrm{MHz}$ etwa dem kürzesten Meßimpuls. Die Frage, wie weit sich Meßimpulse von Störimpulsen trennen lassen, hängt außerdem noch von der Streuung der Amplituden ah, welche beide Sorten von Impulsen, hauptsächlich infolge der Statistik des Verstärkungsvorganges im Vervielfacher, erleiden ${ }^{\mathbf{1 8}}$. Diese Frage wird später behandelt werden. Der Ausgang des Verstärkers enthält entweder eine Endstufe zum Anschluß an einen Oszillographen hoher Schreibgeschwindigkeit, oder eine Diskriminatorstufe zur Aussonderung von Impulsen bestimmter Amplitude. Für höhere Impulszahlen wurde ein entsprechender Untersetzer benutzt.

Als Strahlungsquellen standen für $\alpha-\beta$ - und $\gamma$ Strahlen einige Radiumpräparate zur Verfügung. Zur Untersuchung einer einheitlichen $\alpha$-Strahlung diente ein Polonium-Präparat.

${ }^{18}$ F. S a u te r, Z. Naturforschg. 4a, 682 [1949]. 
Untersuchung der Eigenschaften von Naphthalin-und AnthracenLe uchtschirmen

Wie schon oben erwähnt, eignen sich die Zinksulfid-Leuchtschirme wenig zur Zählung von $\beta$ und $\gamma$-Strahlen. Weit bessere Resultate lassen sich mit Naphthalin- und Anthracen-Leuchtschirmen erzielen, die nach dem oben angegebenen Verfahren hergestellt werden. Diese Leuchtschirme sind bei den verwendeten Dicken von 2 bis $3 \mathrm{~mm}$ für sichtbares Licht nur noch schwach durchscheinend. Ein klar durchsichtiger, aus dem Schmelzfluß auskristallisierter Naphthalinkristall zeigt

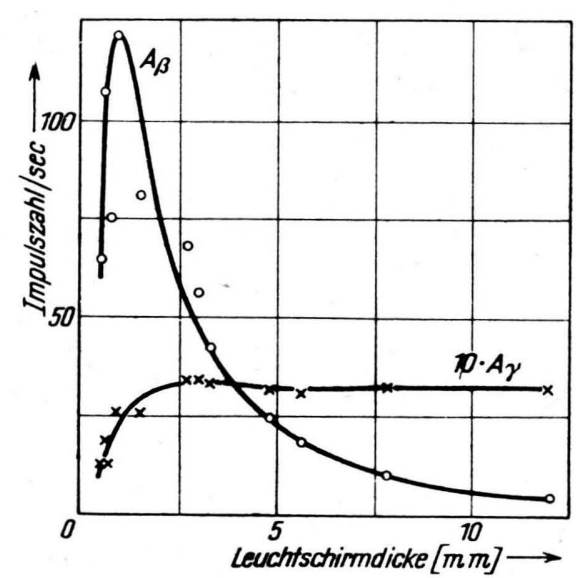

Abb. 2. Impulszahlen in Abhängigkeit von der durchstrahlten Leuchtschirmdicke für Anthracen bei Bestrahlung mit Radium- $\beta$ - und $-\gamma$-Strahlung.

merkwürdigerweise eine wesentlich geringere Empfindlichkeit. Die Untersuchungen über das beste Herstellungsverfahren für Leuchtschirme sind jedoch noch zu wenig ausführlich, um dariiber sichere Aussagen machen zu können. Auch die in der Literatur vorhandenen Angaben sind teilweise noch widersprechend. Es ist jedoch wahrscheinlich, daß auf diesem Gebiet noch wesentliche Verbesserungen $\mathrm{zu}$ erwarten sind. Für die nach dem obigen Verfahren hergestellten Leuchtschirme wurde die für eine bestimmte Strahlungsart günstigste Schichtdicke bestimmt. $\mathrm{Zu}$ diesem $\mathrm{Zweck}$ wurde ëine größere Anzahl von Naphthalin- und Anthracen-Leuchtschirmen verschiedener Dicke hergestellt. Bei Bestrahlung mit den inhomogenen $\beta$ - und $\gamma$-Strahlen eines RadiumPräparates ergaben sich für die gezählten Impulszahlen in Abhängigkeit von der Dicke Kurven, wie sie für Anthracen in Abb. 2 zu sehen sind.
Die Kurve $A_{\beta}$ ist mit $\beta$-Strahlen, die Kurve $A_{\gamma}$ mit $\gamma$-Strahlen gemessen worden. Außerdem sind der besseren Darstellung wegen die Ordinaten der Kurve $A_{\gamma}$ mit dem Faktor 10 multipliziert worden $\left(10 \cdot A_{\gamma}\right)$. Für die Messung beider Kurven befand sich das Radium-Präparat in gleichem Abstand vom Leuchtschirm. Bei der Messung mit $\gamma$-Strahlen wurde die $\beta$-Strahlung des Präparates durch $1 \mathrm{~mm}$ Blei absorbiert. Zur Messung der $\beta$-Strahlung wurde in Ermangelung eines reinen $\beta$-Strahlers die Gesamtstrahlung des Radium-Präparates ohne jede Filterung benutzt, so daß also auch die $\gamma$-Strahlung mitgemessen wurde. Da der Anteil der gemessenen $\gamma$-Strahlen wie aus der $A_{\gamma}$-Kurve hervorgeht, nach Filterung durch $1 \mathrm{~mm}$ Blei nur etwa 1\% der Gesamtstrahlung ausmacht, wurde dieser Fehler in Kauf genommen. Die Kurven zeigen einen ganz ähnlichen Verlauf, wie er auch schon von $\mathrm{K}$ a $1 \mathrm{~lm}$ a n $\mathrm{n}^{12,13}$ gefunden wurde. Für die $\beta$-Strahlung ergibt sich bei Anthracen ein Maximum bei etwa $1 \mathrm{~mm}$ Dicke und anschließend ein durch die Absorption der $\beta$-Strahlung im Anthracen hervorgerufener Abfall. Bei der Messung von $\gamma$-Strahlung liegt das Maximum bei etwa $2,5 \mathrm{~mm}$, entsprechend der Schichtdicke, aus der noch Fluoreszenzstrahlung austreten kann. Anschließend ergibt sich ein geradliniger Verlauf, da bis zu Schichtdicken von $10 \mathrm{~mm}$ die Absorption der $\gamma$-Strahlung im Anthracen noch nicht merklich wird. Qualitativ ist der Verlauf der Kurven für Naphthalin ebenso wie für Anthracen. Die maximale Dicke, aus der noch Fluoreszenzstrahlung austreten kann, ist dagegen etwas größer, sie beträgt 3 bis $4 \mathrm{~mm}$.

Um einen Vergleich der Empfindlichkeit von Anthracen und Naphthalin zu bekommen, wurden sämtliche Kurven unter gleichen Bedingungen aufgenommen. Abb. 3 zeigt diese Kurven im gleichen Maßstab, wobei $\mathrm{A}_{\beta}$ die Kurve für AnthracenLeuchtschirm bestrahlt mit $\beta$-Strahlung, $\mathrm{A}_{\gamma}$ bestrahlt mit $\gamma$-Strahlung usw. bedeutet. Aus den Kurven ergibt sich, daß Anthracen-Leuchtschirme sowohl für $\beta$ - wie für $\gamma$-Strahlen etwa 10 -fach höhere Impulszahlen geben als Naphthalin-Leuchtschirme. Wie später noch weiter auseinandergesetzt wird, streuen die Amplituden dieser Impulse über einen außerordentlich großen Bereich. Man kann dabei ohne weiteres weder eine obere, noch eine untere Grenze definieren. Bei den Messungen werden deshalb mit Hilfe der Diskriminatorstufe des Verstärkers diejenigen Impulse 
gezählt, die eine bestimmte Mindestamplitude überschreiten. Diese Mindestamplitude ist dadurch festgelegt, daß die durch thermische Emission erzeugten Impulse, der Nulleffekt, noch zu vernachlässigen ist, oder doch mindestens ohne unzulässige Beeinträchtigung der Meßgenauigkeit abgezogen werden kann. Die Mindestamplitude war bei den Messungen für Anthracen und Naphthalin gleich gewählt worden. Da Anthracen dabei 10-mal mehr Impulse liefert als Naphthalin, sind also auch die Amplituden der von Anthracen ge-

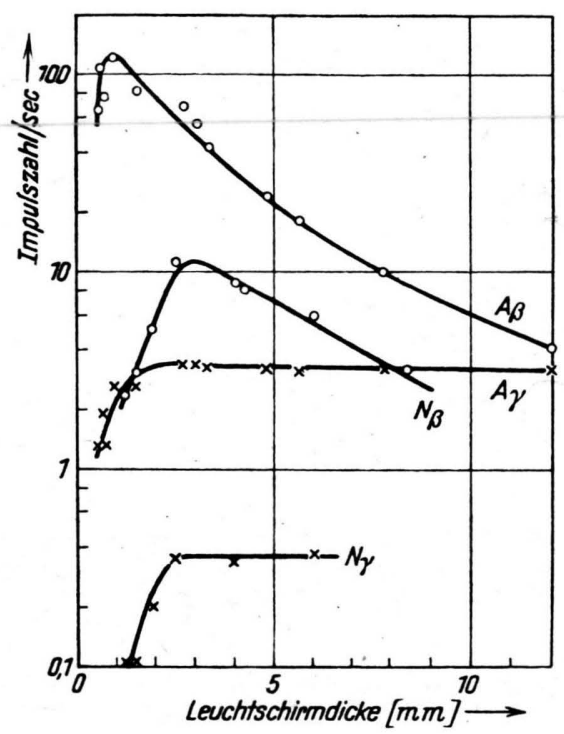

Abb. 3. Vergleich der Ausbeute von Anthracen- und Naphthalin-Leuchtschirmen, bestrahlt mit Radium- $\beta$ und $-\gamma$-Strahlen.

lieferten Impulse im Mittel wesentlich größer als diejenigen von Naphthalin. Über das wirkliche Intensitätsverhältnis der Fluoreszenzstrahlung von Anthracen und Naphthalin sagt dieses Ergebnis nichts aus, da es durch die verschiedene Lage der Fluoreszenzbanden der beiden Substanzen relativ zum spektralen Empfindlichkeitsbereich des Vervielfachers bestimmt ist. Die Fluoreszenzbanden des Naphthalins liegen bei etwa 3200 bis $3600 \AA$ A diejenigen des Anthracens bei 4000 bis 4800 A. Das Maximum der Photoschicht des Vervielfachers I P 21 liegt bei $4200 \AA$. Da die Glaswand des Vervielfachers das UV-Licht weitgehend absorbiert, so wird Naphthalin bei diesen Messungen stark benachteiligt. Die Verwendung des UV-durchlässigen Vervielfachers I P 28 brachte deswegen keinen Fortschritt, weil er bei wesent- lich größerem Dunkelstrom eine 10-fach geringere Verstärkung als I P 21 besitzt. Als günstigste Kombination ergab sich somit der Vervielfacher I P 21 mit Anthracen-Leuchtschirm.

I mpulsdauer und Impulsform

Ein einzelnes Elektron, welches etwa durch Photoeffekt an der Photokathode eines Vervielfachers ausgelöst und dann verstärkt wird, ergibt einen Impuls am Ausgang des Vervielfachers, dessen Dauer durch die verschiedenen Laufzeiten der Sekundärelektronen gegeben ist. Diese Impulsdauer hängt also wesentlich von der Bauart des Vervielfachers ab. Von R. D. Sard ${ }^{19}$ wurde für den Typ 931 A eine entsprechende Rechnung durchgeführt. Die Impulsdauer wurde zu $6 \cdot 10^{-10} \mathrm{sec}$ gefunden, was auch mit anderen Messungen ungefähr übereinstimmt. Eine direkte oszillographische Aufnahme derartig kurzer Impulse läßt sich zwar nicht durchführen, doch ist die Bestimmung der Impulsdauer auf indirekte Weise, z. B. mit der von $\mathrm{B}$ a $\mathrm{y}$ und $\mathrm{P}$ a $\mathrm{p} \mathrm{p}^{6}$ benutzten Koinzidenzmethode, möglich. Die Lichtblitze, welche eine im Leuchtschirm absorbierte Strahlung erzeugt, geben bei guter optischer Ausnutzung wesentlich mehr als ein Elektron aus der Photokathode des Vervielfachers. Außerdem ist der zeitliche Verlauf dieses Prozesses bestimmt durch den Abklingungsvorgang des benutzten angeregten Leuchtstoffes. Die erhaltenen Impulse sind infolgedessen von wesentlich längerer Dauer, so daß eine direkte oszillographische Messung möglich erscheint. Mit dem oben beschriebenen Verstärker wurden solche Aufnahmen für Zinksulfid-, Anthracen- und Naphthalin-Leuchtschirme durchgeführt, wobei die kürzesten Impulse allerdings schon die obere Frequenzgrenze des Verstärkers erreichen, so daß eine gewisse Verfälschung auftritt. Die Ankopplung des Vervielfachers an den Verstärker geschah mit einem 100-Ohm-Anodenwiderstand, an dem Impulse von größenordnungsmäßig $10^{-2}$ bis $10^{-3} \mathrm{~V}$ auftraten. Mit einer Gesamtverstärkung von etwa $10^{4}$ wurden dann Amplituden bis zu etwa $100 \mathrm{~V}$ erreicht. Diese hohen Amplituden wurden infolge der relativ geringen Empfindlichkeit des zur Aufzeichnung benutzten HochleistungsOszillographen nötig. Die außerordentlich hohe Schreibgeschwindigkeit, welche zur photographischen Registrierung der kürzesten Impulse nötig

19 R. D. S a r d, J. appl. Physies 17, 768 [1946]. 

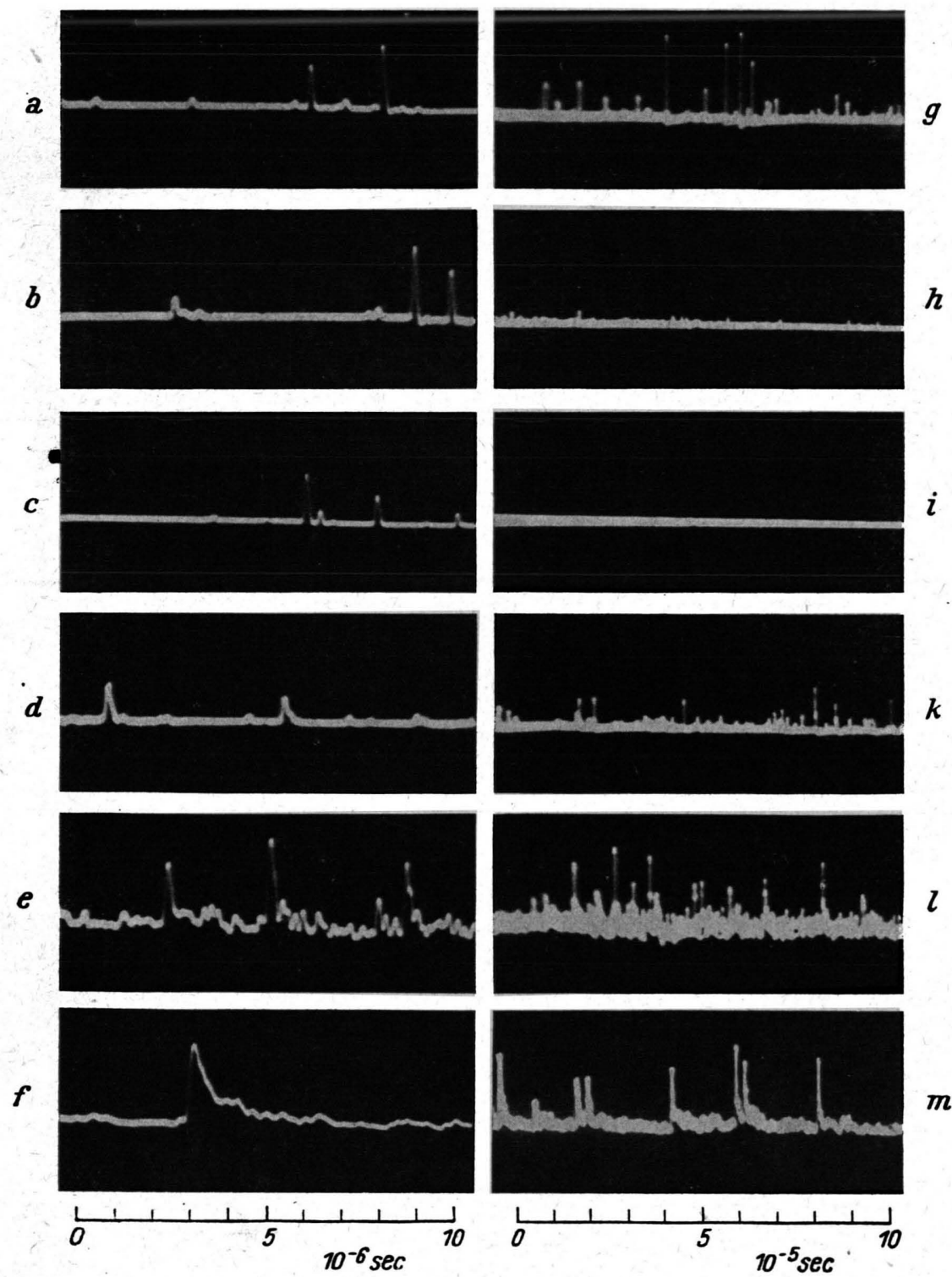

Abb. 4. Impulse von Anthracen-, Naphthalin- und Zinksulfid-Leuchtschirmen, bestrahlt mit $\alpha$-, $\beta$ - und $\gamma$-Strah len. a, b, c, g Anthracen-Leuchtschirm $(\beta-\gamma$-Strahlung); d, k Naphthalin-Leuchtschirm $(\beta$ - $\gamma$-Strahlung $)$; e, f, 1, m Zinksulfid-Leuchtschirm ( $\alpha$-Strahlung); h ohne Leuchtschirm mit $\beta$ - und $\gamma$-Strahlung; i mit Anthracen ohne Strahlungsquelle.

war, konnte mit dem AEG-Rohr H R P 1/100/15 bei $10 \mathrm{KV}$ Beschleunigungsspannung bequem erreicht werden.

Einige typische Oszillogramme sind in Abb. 4 wiedergegeben. Abb. $4 \mathrm{a}-\mathrm{c}$ zeigt die Impulse eines Anthracen-Leuchtschirmes, bestrahlt mit Radium$\gamma$-Strahlung. Aus dem beigegebenen Zeitmaßstab läßt sich ablesen, daß die Impulsdauer etwa $1^{-7}$ sec beträgt. Auffallend ist die schon früher erwähnte Streuung der Amplituden. Sie wird noch deutlicher erkennbar an Aufnahmen mit langsamerer Zeitlinie (Abb.4g). Es ist nun wichtig $\mathrm{zu}$ wissen, welche von diesen Impulsen durch Szintillationen im Leuchtschirm erzeugt worden sind. $\mathrm{Zu}$ diesem $\mathrm{Z}$ weck führt man zwei Differenzmessungen aus. Zuerst entfernt man den Leuchtschirm, läßt jedoch die $\gamma$-Strahlung des Radiumpräparates weiterhin auf den Vervielfacher fallen. 
Man erhält Oszillogramme entspr. Abb. 4h, welche eine Menge sehr klęner Impulse zeigen. Entfernt man dagegen die Strahlungsquelle und läßt dẹ Leuchtschirm am Vervielfacher, so ist, wie Abb. $4 \mathrm{i}$ zeigt, bei der vorliegenden Meßempfindlichkeit überhaupt kein Impuls zu bemerken. Die Versuche ergeben also, daß die in Abb. $4 \mathrm{~h}$ sichtbaren Impulse durch die $\gamma$-Strahlung direkt an den Elektroden des Vervielfachers ausgelöst worden sind. Dagegen sind die von thermischen Elektronen herrührenden Impulse im Mittel wesentlich kleiner, so daß sie in Abb.4i nicht mehr erkennbar sind. Die durch $\gamma$-Strahlen erzeugten Impulse sind deswegen größer, weil ein $\gamma$-Quant im Mittel mehr als ein Elektron im Vervielfacher auslöst. Dies geht z. B. aus den Amplitudenverteilungskurven hervor, welche von Meyer, Huber und Baldinger ${ }^{5}$ für Licht- und $\gamma$-Strahlen gemessen wurden. Da die durchdringende $\gamma$-Strahlung nicht nur in der Photokathode, sondern auch in den folgenden Stufen des Vervielfachers Elektronen auslösen kann, liefert sie eine Menge Impulse sehr kleiner Amplitude, die insbesondere bei Messungen der Amplitudenverteilung störend sein können und durch Differenzmessungen beseitigt werden müssen. $Z u$ diesem $Z$ weck kann man außer mit Hilfe der obigen Methode in manchen Fällen auch die Differenz der Impulszahlen ermitteln, die bei normalem Betrieb des Vervielfachers auftreten und bei Spannungsdifferenz Null zwischen bestrahlter und nächstfolgender Elektrode ${ }^{5}$.

Einen Vergleich der Eigenschaften verschiedener Leuchtschirmsubstanzen geben die folgenden Oszillogramme. Unter den gleichen Bedingungen, insbesondere gleicher Bestrahlung und gleicher Verstärkung, wurden die in Abb. $4 \mathrm{~d}$ und $4 \mathrm{k}$ wiedergegebenen Impulse eines $2.5 \mathrm{~mm}$ dicken Naphthalin-Leuchtschirmes aufgenommen. Die Impulsdauer ist etwa 2-mal so lang wie bei Anthracen. Außerdem zeigt insbesondere der Vergleich von Abb. $4 \mathrm{~g}$ und $4 \mathrm{k}$ deutlich, daß die Amplituden bei Naphthalin im Mittel wesentlich kleiner sind als bei Anthracen. Zur Zählung von $\alpha$-Strahlen wurden Zinksulfid-Schirme verschiedener Nachleuchtdauer benutzt. Abb. 4 e und 41 zeigen Impulse von $\alpha$-Strahlen mit schneller und langsamer Zeitlinie für einen sehr kurz nachleuchtenden, Abb. $4 \mathrm{f}$ und $4 \mathrm{~m}$ für einen länger nachleuchtenden Zinksulfid-Schirm. Die Impulsdauer beträgt im einen Fall etwa $3 \cdot 10^{-7} \mathrm{sec}$, im anderen Fall etwa $1 \cdot 10^{-6}$ sec. Auch beim Zinksulfid ist die starke Streuung der Amplituden vorhanden. Wie vorher läßt sich durch eine Differenzmessung die Zahl der $\alpha$-Strahlen allein ermitteln. Wird der Zinksulfid-Schirm nicht bestrahlt, so erhält man das gleiche Bild wie in Abb. 4i, d. h. keine sichtbaren Impulse. Läßt man die $\beta$ - und $\gamma$-Strahlung auf den Leuchtschirm auffallen, absorbiert jedoch die $\alpha$-Strahlung vor Erreichen des Schirmes, so erhält man ein Abb. 4h ähnliches Bild, d. h. eine Reihe sehr kleiner Impulse, die durch $\gamma$-Strahlen im Vervielfacher selbst erzeugt worden sind. Die durch $\beta$ - und $\gamma$-Strahlen im Zinksulfid erzeugten Impulse können nur sehr unwesentlich dabei beteiligt sein. Eine weitgehende Trennung von $\alpha$-Strahlen einerseits, $\beta$ - und $\gamma$ Strahlung andererseits ist also durch Wahl der entsprechenden Leuchtschirme leicht möglich, da nämlich $\alpha$-Strahlen in Anthracen und Naphthalin nur außerordentlich kleine Impulse gegenüber $\beta$ und $\gamma$-Strahlung geben.

\section{Amplitudenverteilung}

Ein Geiger-Müller-Zählrohr liefert als Auslösezählrohr unabhängig von der primär erzeugten Ionisation bei konstanter Spannung stets den gleichen Ausgangsimpuls. Beim Proportionalzählrohr dagegen ist dieser Impuls proportionaI der Zahl der primären Ladungsträger. In diesem Sinne ist ein Leuchtschirm-Zähler mit einem Proportionalzählrohr za vergleichen. Die mittlere Größe der Ausgangsimpulse des Vervielfachers ist proportional der Zahl der am Eingang erzeugten Elektronen. Diese wieder sind proportional der Zahl der Lichtquanten, welche vom Leuchtschirm kommt und eine Funktion der darin absorbierten Strahlungsenergie ist. Eine Einschränkung muß dabei allerdings sofort gemacht werden. Die Amplituden eines LeuchtschirmZählers zeigen, wie aus den Oszillogrammen der Abb. 4 anschaulich hervorgeht, eine außerordentlich große Streuung. Diese Streuung existiert auch dann, wenn man mit homogener Strahlung und weitgehend homogenen Leuchtschirmen arbeitet. Diese Tatsache ist von verschiedenen Seiten experimentell gefunden worden. Infolgedessen läßt sich zu einem bestimmten Eingangsimpuls des Zählers kein definierter Wert der Ausgangsamplitude angeben, sondern eine Verteilungsfunktion der Amplitudenwerte. Aus dieser experimentell zu gewinnenden Amplitudenverteilung kann 
man dann, wie aus den folgenden Messungen und insbesondere der anschließenden Arbeit von F. Sa uter ${ }^{18}$ hervorgeht, die Größe des Eingangsimpulses im Prinzip ermitteln. Beim Proportionalzählrohr beobachtet man im allgemeinen keine derartig große Amplitudenstreuung. Die Erklärung ergibt sich aus dem Folgenden und sei hier vorweggenommen. Die Zahl der Elektronen, welche im Eingang des Vervielfachers erzeugt werden, ist meistens um einige Größenordnungen geringer als die Zahl der primären Ladungsträger beim Proportionalzählrohr. Die statistische Streuung beim Verstärkungsprozeß ist infolgedessen beim Vervielfacher wesentlich größer. Bei der gleichen Zahl startender Elektronen dürfte die Streuung in beiden Fällen einen ähnlichen Wert haben. Die Amplitudenverteilung in den oben gezeigten Oszillogrammen hat eine Reihe von Ursachen. Ein experimentell bedingter Grund ist zunächst die nicht ganz zu vermeidende Inhomogenität der Leuchtschirme, außerdem die Inhomogenität der benutzten Strahlungsquelle. Weiterhin kommt jedoch als prinzipiell wichtiger Faktor die Statistik der Lichterzeugung im Leuchtschirm, die Statistik des Photoeffektes und der Verstärkung durch Sekundäremission im Vervielfacher hinzu. Die Statistik des Vervielfachers allein läßt sich am besten dadurch untersuchen, daß man dessen Photokathode mit Licht bestrahlt. Dadurch ist gewährleistet, daß die Vervielfachung mit nur einem Photoelektron beginnt. Die dabei auftretende Amplitudenverteilung ist mehrfach gemessen worden ${ }^{1,5,20}$. Die Ergebnisse weichen voneinander ab, was insofern bedauerlich ist, als die Kenntnis dieser Verteilungsfunktion die Grundlage für eine theoretische Behandlung der Vorgänge bei mehreren startenden Elektronen ist.

Eine kompliziertere Amplitudenverteilung ist für die bereits bei Zimmertemperatur aus den Elektroden des Vervielfachers austretenden thermischen Elektronen zu erwarten, da sie nicht nur an der ersten, sondern auch an den folgenden Stufen entstehen können, wodurch insbesondere die Zahl der Impulse mit kleinen Amplituden erhöht wird. Die von thermischen Elektronen herrührenden Störimpulse überlagern sich den zu messenden Impulsen und begrenzen die Mindestamplitude der Meßimpulse, die noch erfaßt werden kann. Abb. 5 zeigt eine solche Messung. Die

20 G. A. Morton u. I. A. It it c h e ll, Nucleonics 4, 16 [1949].
Verteilungskurven sind so gewonnen, daß für je eine bestimmte, am Diskriminator eingestellte Amplitude in Volt sämtliche Amplituden größer und gleich dieser Amplitude gezählt werden. Man erhält damit eine Art Integralkurve der Amplitudenverteilung. Die obere Kurve der Abb.5 ist zum Vergleich eine Verteilungskurve für Radium$\gamma$-Strahlung und Naphthalin-Leuchtschirm. Die untere Kurve der durch thermische Elektronen erzeugten Störimpulse zeigt einen wesentlich steileren Abfall, so daß eine genügende Trennung von Meß- und Störimpulsen bei der vorliegenden

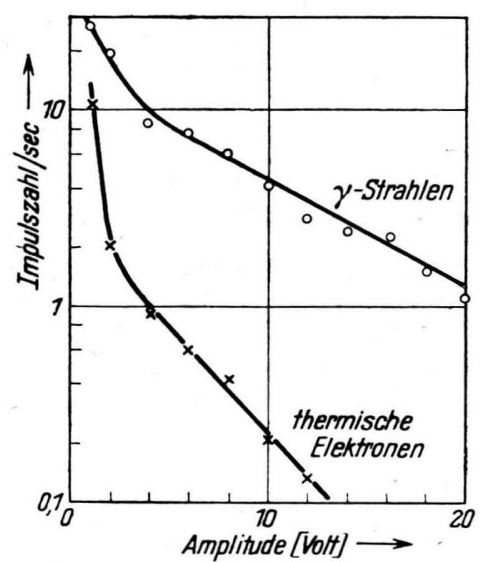

Abb. 5. Amplitudenverteilung für thermische Elektronen und $\gamma$-Strahlung mit Naphthalin-Leuchtschirm.

hohen Intensität von $\gamma$-Strahlen möglich ist. Ein einheitlicher Verlauf der Kurven existiert nicht und ist nach dem oben Gesagten auch nicht zu erwarten. Die Messung erfolgte bei $20^{\circ} \mathrm{C}$ mit I P 21 bei $100 \mathrm{~V} /$ Stufe. Bei kleinen Intensitäten der zu messenden Strahlung ist der vorliegende Störpegel bereits zu.hoch. Außerdem ist aus der Verteilungskurve für $\gamma$-Strahlen ersichtlich, daß die Ausbeute an gezählten $\gamma$-Strahlen um so größer ist, je kleinere Amplituden noch gemessen werden können. Da diese Mindestamplitude nur durch die Zahl der Störimpulse begrenzt wird, müssen diese soweit wie möglich beseitigt werden. Ein Weg dazu ist die Kühlung des Vervielfachers. Bei der Temperatur der flüssigen Luft verschwindet die in Abb. 5 gezeigte Kurve der Störimpulse vollständig, und die $\gamma$-Kurve kann bis zu außerordentlich kleinen Amplituden herab gemessen werden. Eine weitere Methode ist die Benutzung zweier Vervielfacher mit einem ge- 
meinsamen Leuchtschirm. Siebt man mit einem Koinzidenzverstärker nur die koinzidenten Ausgangsimpulse der beiden Vervielfacher entsprechend den Leuchtschirmszintillationen aus, so fallen die thermisch bedingten Störimpulse weg. Dieses Verfahren ist in manchen Fällen bequemer als die lästige Kühlung des Vervielfachers.

Die Amplitudenverteilung des Leuchtschirmzählers ist dadurch bedingt, daß zur Statistik des Vervielfachers diejenige des Leuchtschirmprozesses hinzukommt. Um einigermaßen einfache

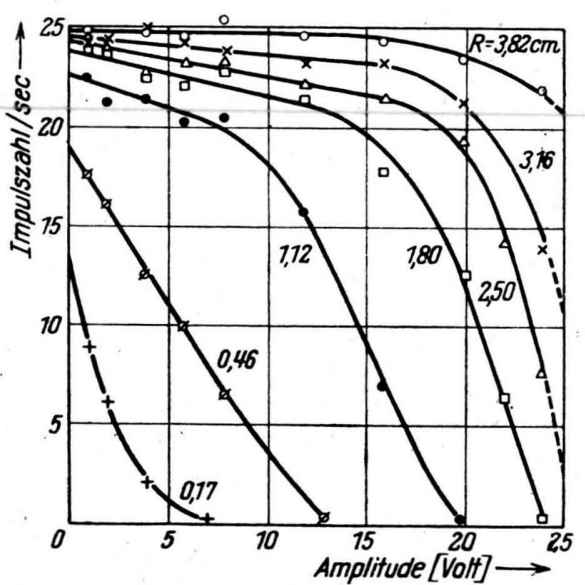

Abb. 6. Amplitudenverteilung für Polonium- $\alpha$-Strahlung mit Zinksulfidschirm bei verschiedener Restreichweite.

Verhältnisse zu erhalten, wurde deswegen zunächst mit homogener Strahlung und möglichst homogenem Leuchtschirm gearbeitet. Als Strahlenquelle stand die weitgehend homogene $\alpha$-Strahlung eines, Polonium-Präparates zur Verfügung. Als Nachweismittel wurde ein möglichst sorgfältig hergestellter Zinksulfid-Schirm benutzt. Die Meßanordnung ist aus Abb.1 a zu ersehen. Zur meßbaren Änderung der Energie der auf den Leuchtschirm auffallenden $\alpha$-Strahlen wurde der Luftdruck zwischen Präparat und Leuchtschirm geändert. Das Ergebnis zeigen die Verteilungskurven der Abb.6. Es sind Integralkurven, die nach der gleichen Methode gewonnen wurden wie bei Abb.5. Der Parameter der einzelnen Kurven ist die beim Auftreffen auf dem Leuchtschirm noch vorhandene Restreichweite, also eine Funktion der im Leuchtschirm absorbierten Energie. Von den eingezeichneten Meßpunkten sind hier, wie auch bei allen folgenden Kurven, die Störimpulse bereits abgezogen. Bei den größten Rest- reichweiten haben die Verteilungsfunktionen einen annähernd horizontalen Ast. Die an dieser Stelle gezählte Impulszahl entspricht $100 \%$ der auffallenden Zahl von $\alpha$-Strahlen, was durch Vergleichsmessung mit einem Zählrohr nachgeprüft wurde. Aus den Daten der Apparatur läßt sich berechnen, daß im Mittel in diesem Fall etwa 100 Photoelektronen im Eingang des Vervielfachers gestartet sind. Die Streuing der Verteilungskurven ist deshalb gering. Mit abnehmender Restreichweite nimmt die Streuung zu und die Zahl der gezählten $\alpha$-Strahlen ab. Bei $0,46 \mathrm{~cm}$ Restreichweite werden nur noch etwa $70 \%$ der $\alpha$-Strahlen gezählt. Diese Werte sind natürlich

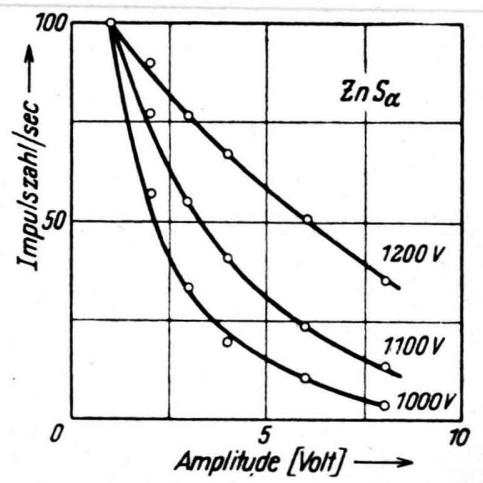

Abb. 7. Amplitudenverteilung für $\alpha$-Strahlen mit Zinksulfid in Abhängigkeit von der am Vervielfacher liegenden Spannung.

nur für die hier gewählten Versuchsbedingungen maßgebend. Gelingt es, die pro $\alpha$-Strahl erzeugte Zahl von Photoelektronen zu erhöhen, so läßt sich auch bei kleinerer Restreichweite quantitativ zählen. Dazu muß die Ausbeute des Leuchtschirmes, die optische Ausnutzung des Fluoreszenzlichtes und die Empfindlichkeit der Photokathode soweit wie möglich erhöht'werden.

Es ist zu erwarten, daß die Verteilungsfunktion auch rom Verstärkungsfaktor des Vervielfachers, d. h. von der an ihm liegenden Spannung. abhängen wird. Abb. 7 zeigt Verteilungskurven für $\alpha$-Strahlen und Zinksulfid-Schirm bei verschiedenen Spannungen am Vervielfacher. Es ergibt sich, daß mit zunehmender Spannung der Abfall der Kurven flacher wird, was einen erhöhten Wirkungsgrad des Zählers bedeutet. Gegenüber Abb. 6 ist hierbei mit $\alpha$-Strahlen geringerer Reichweite gearbeitet worden. Bei dieser und den folgenden Messungen wurde außerdem kein Hohlspiegel hinter dem Leuchtschirm benutzt, wo- 
durch natürlich der Wirkungsgrad absinkt. Außerdem wurden sämtliche Verteilungskurven für eine Mindestamplitude auf gleiche Impulszahl pro Sekunde reduziert. Diese Mindestamplitude ist durch den jeweils vorhandenen Störpegel vorgegeben. Der Abfall der Verteilungsfunktion zu größeren Amplituden hin ist dann als Maß für den Wirkungsgrad der betreffenden Anordnung $\mathrm{zu}$ betrachten. Abb. 8 zeigt Verteilungskurven für Zinksulfid, bestrahlt mit der inhomogenen $\alpha$ Strahlung eines Radium-Präparates, Anthracen mit Radium- $\beta$ - und $\gamma$-Strahlung und Naphthalin mit Radium- $\beta$-Strahlung. Der Wirkungsgrad, d. h. die Zahl der im Mittel erzeugten 'Photoelektronen

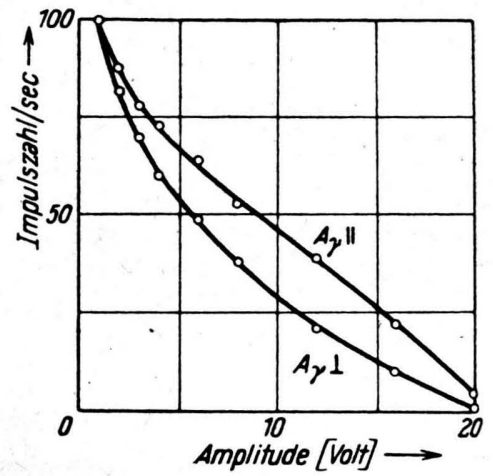

Abb. 9. Abhängigkeit der Amplitudenverteilung vom Einfallswinkel zum Leuchtschirm.

im Vervielfacher ist am größten bei $\alpha$-Strahlung und Zinksulfid, geringer bei $\beta$-Strahlung und Anthracen, und noch kleiner für Anthracen und $\gamma$ Strahlung. Naphthalin-Leuchtschirme sind wesentlich weniger empfindlich, wie sich aus der mit $\beta$ Strahlung gewonnenen Kurve ergibt. Dies bestätigt ein bei der Untersuchung der Dickenabhängigkeit der Leuchtschirme bereits gefundenes Ergebnis. Bei Anderung der Energie der einfallenden Strahlung erhält man ebenfalls eine Änderung der Verteilungsfunktion. Filtert man die weichen Komponenten der $\gamma$-Strahlung aus, so erhält man mit der so gewonnenen energiereicheren Strahlung einen flacheren Abfall als mit weicher Strahlung.

Wesentlich ist auch die Einfallsrichtung des $\gamma$-Strahls relativ zum Leuchtschirm. Abb. 9 zeigt an zwei Verteilungskurven ein Beispiel dafür. Fällt der ausgeblendete $\gamma$-Strahl senkrecht auf die Oberfläche des Leuchtschirmes in Richtung der Photokathode des Vervielfachers (Abb. 1b), so er- gibt sich die Kurve $A_{\gamma} \perp$ mit steilem Abfall. Fällt der $\gamma$-Strahl parallel zu dieser Richtung ein, also parallel zur Photokathode, so durchstrahlt er eine größere Schichtdicke des Leuchtschirmes. Für die erzeugte Fluoreszenzstrahlung ist jedoch die im Leuchtschirm zurückzulegende Strecke, also die Absorption, nicht größer als im vorigen Fall. Die Kurve $A_{\gamma}$ ll zeigt also einen flacheren Verlauf, entspr. einem höheren Wirkungsgrad.

Zusammenfassend kann man also sagen, daß die Form der Verteilungskurven bei sonst gleichbleibenden Bedingungen von der Zahl der im Vervielfacher startenden Elektronen abhängig ist.

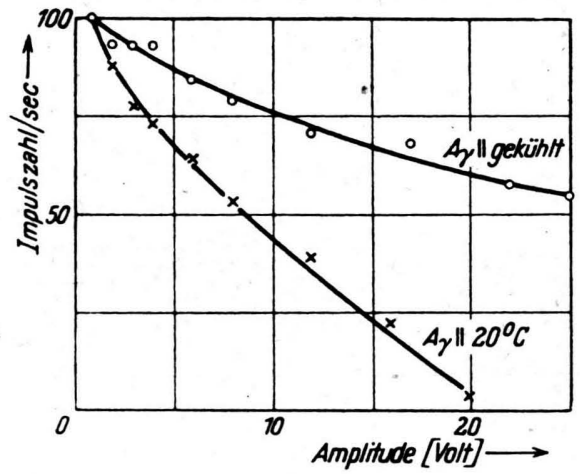

Abb. 10. Amplitudenverteilung bei Kühlung des Vervielfachers mit flüssiger Luft und bei Zimmertemperatur. Der Verstärkungsfaktor ist in beiden Fällen verschieden.

Läßt sich aus theoretischen Betrachtungen an Hand der gemessenen Verteilungsfunktion die Zahl dieser Primärelektronen ermitteln, so kann man damit einen weiteren Schluß auf die im Leuchtschirm absorbierte Energie der zu messenden Strahlen ziehen. In diesem Sinne gestattet also der Leuchtschirm-Zähler nicht. nur die Zählung von Impulsen, sondern im Prinzip auch eine Energiemessung.

Wirkungsgrad im Vergleich zum Geiger-M üller-Zählrohr

Wie schon erwähnt, werden $\alpha$-Strahlen einer bestimmten Mindestenergie mit 100\% Wirkungsgrad gezählt. Für Radium- $\gamma$-Strahlung und AnthracenLeuchtschirm ergibt ein Vergleich mit einem Geiger-Müller-Zählrohr bei gleichem bestrahltem Querschnitt den doppelten Wirkungsgrad für den Leuchtschirm-Zähler. Dies gilt für eine Temperatur von $20^{\circ} \mathrm{C}$, wo die noch meßbare Mindest- 
amplitude durch thermische Störimpulse gegeben ist. Unterdrückt man die thermische Emission

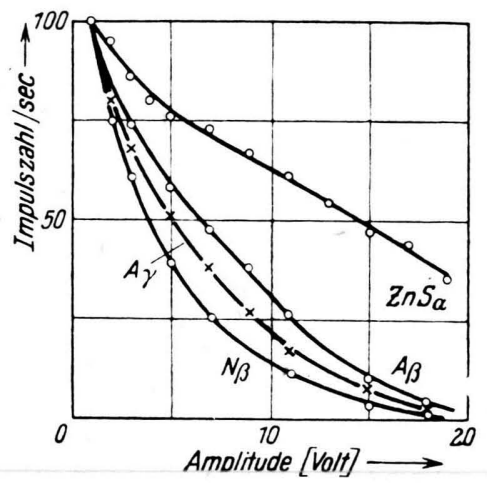

Mh..8. Vergleich des Wirkungsgrades an Hand der Amplitudenverteilung für $\alpha$-Strahlen mit Zinksulfid, $\beta$ - und $\gamma$-Strahlen mif Anthracen, $\beta$-Strahlen mit Naphthalin. durch Kühlung mit flüssiger Luft, so läßt sich der Verstärkungsfaktor wesentlich erhöhen, und man erhält einen bedeutend höheren Wirkungsgrad. Die entsprechende Verteilungsfunktion (Abb.10) zeigt gegenüber der bei $20^{\circ} \mathrm{C}$ gemessenen Kurve einen dementsprechend flacheren Abfall. Man bekommt etwa die 8 - bis 10 -fache Impulszahl/sec bei gleicher $\gamma$-Strahlung, verglichen mit dem Zählrohr und reduziert auf gleichen bestrahlten Querschnitt.

A n m. b. d. K o r r.: Während der Drucklegung der vorliegenden Arbeit erschien eine größere Zahl von Arbeiten, die sich mit dem gleichen Thema beschäftigen. Die Ergebnisse sind im wesentlichen in Übereinstimmung mit den hier gefunden. Eine zusammenfassende Darstellung und eine ausführliche Literaturzusammenstellung finden sich bei W. H. Jordan und P. R. B e ll, Nucleonics 5, Nr. 4, 30 [1949].

\title{
Zur Statistik bei Elektronenvervielfachern
}

\author{
Von Fritz Sauter: \\ (Z. Naturforschg. 4 a, 682-691 [1949]; eingegangen am 4. März 1949)
}

\begin{abstract}
Unter der Annahme, daß der Verstärkungsgrad eines Vervielfachers in seinen Schwankungen einem bestimmten Wahrscheinlichkeitsgesetz unterworfen ist, kann man einen allgemeinen Zusammenhang aufstellen zwischen den Amplitudenverteilungskurven der Eingangs- und Ausgangsimpulse. Für den Verstärkungsgrad wird aus den Experimenten eine exponentielle Verteilungsfunktion erschlossen. Ihre Anwendung auf verschiedene konkrete Probleme führt auf Amplitudenverteilungskurven für die Ausgangsimpulse, welche in Parallele zu verschiedenen gemessenen Verteilungskurven gebracht werden können.
\end{abstract}

\section{Problemstellung}

$\mathrm{D}$ ie folgenden Ausführungen beschäftigen sich mit einer Klasse von Verstärkern, deren wichtigster Vertreter der Sekundärelektronen-Vervielfacher (Multiplier) ist, und zu der u. a. auch das Proportionalzählrohr gehört. Sie sind dadurch charakterisiert, daß sie, im Gegensatz etwa zu den Auslösezählrohren oder ähnlichen Vorrichtungen, nicht nur einzelne Impulse zu registrieren gestatten, sondern diese auch so verstärken, daß die mittlere Stärke der Endimpulse proportional ist zur mittleren Stärke der Eingangsimpulse.

Nun erfolgt diese Vervielfachung allerdings keineswegs stets im gleichen Verhältnis; vielmehr bekommt man auch bei gleichbleibender Art und Stärke der Eingangsimpulse am Ausgang des Vervielfachers Impulse der verschiedensten Stärke mit beträchtlicher Streuung um den Mittelwert. Über diese durch den Vervielfacher bedingte
Streuung der Endimpulse überlagert sich noch eine weitere Streuung infolge der im allgemeinen vorhandenen Schwankungen in der Stärke der Eingangsimpulse, so daß man experimentell Verteilungskurven für die Stärken der Endimpulse findet, die in ihrer Gestalt meist wesentlich von der Art der die einzelnen Impulse auslösenden Vorgänge auf der Eingangsseite des Vervie]fachers abhängen.

Über die Entstehung und die Deutung dieser Amplitudenverteilungskurven scheint in der Literatur $^{1}$ vorerst noch nicht völlige Klarheit zu bestehen. Wenn auch im letzten Jahr an verschiedenen Stellen theoretische Betrachtungen zu dem hier behandelten Problem veröffentlicht wur-

* (20 b) Göttingen, Calsowstr. 7 .

1 Vgl. die ausfiihrliche Literaturzusammenstellung in der Arbeit von E. F ü n f e r, Zählung von Elementarteilchen und Quanten mit Elektronenvervielfacher und Leuchtschirm. Z. Naturforschg. 4a, 672 [1949]. 